\title{
COVID-19 Publication and the Future of Research
}

\author{
Seraph Shi Kei Wu ${ }^{1}$, Sunny Chi Lik $\mathrm{Au}^{2^{*}}$ \\ ${ }^{1}$ LKS Faculty of Medicine, University of Hong Kong, Hong Kong \\ ${ }^{2}$ Department of Ophthalmology, Pamela Youde Nethersole Eastern Hospital, Tung Wah Eastern Hospital, Hong \\ Kong \\ *Corresponding Author: Sunny Chi Lik Au, Department of Ophthalmology, Pamela Youde Nethersole Eastern \\ Hospital, Tung Wah Eastern Hospital, Hong Kong; Email: kilihcua@gmail.com
}

Received Date: 20-07-2021; Accepted Date: 06-08-2021; Published Date: 13-08-2021

Copyright $^{\odot} 2021$ by Au SCL, et al. All rights reserved. This is an open access article distributed under the terms of the Creative Commons Attribution License, which permits unrestricted use, distribution, and reproduction in any medium, provided the original author and source are credited.

\section{Letter to Editor}

It has been more than 1 year since the COVID-19 pandemic despite the advance in medicine and healthcare, human appears small in front of the frequent emerging infectious diseases in recent decades [1]. These are partly caused by the raise in microbial adaptation, human activity, and substantial international movement. A global pandemic with multi places lockdown affected our way of living and inevitably the way of conducting academic studies and researches. It has significantly delayed recruitment in clinical research trials, and potentially gapping the development of science. Translational research took up the leading role in converting the scientific findings into practical health care provision [2]. Practitioners played the intimate role in collaborating across research centers and hospitals where COVID-19 patients were hospitalized. During the COVID-19 pandemic, many innovative advances emerged such as telemedicine, teleconsultation, virtual webinar or even conferences were blooming in the healthcare sector [3,4]. We should all grasp this golden opportunities to cooperate at the national and international level, which the evolvement of the Journal of Ophthalmology and Advance Research is one of the many examples among all.

Few fundamental obstacles in research still got to be sort out, including efficacy of recruitment, reaching out to potential participants, validating the results, and dissemination of scientifically true findings. By the time of pandemic, numerous reports have been published online or were submitted to preprint servers [5]. New pre-print platforms were even opened particularly to cater COVID-19 materials. Worldwide researchers invested their time, and spent their efforts on their respective fields to carry out their scientific work across different disciplines like

Wu SSK | Volume 2; Issue 2 (2021) | JOAR-2(2)-019 | Letter to Editor

Citation: Au SCL, et al. COVID-19 Publication and the Future of Research. J Ophthalmol Adv Res. 2021;2(2):1-3. 
ocular biochemistry, virology, immunology, and pathology. Ophthalmologists were also working hard on ocular disease transmission, infection control practices, experimental treatment, and even in post-vaccine era [6]. An exponential increase in scientific manuscripts has been collected in several databases about COVID-19. Scientists, researchers and publishers collaborated to mobilize sharing of resources and build the evidence-based practices addressing the COVID-19 pandemic under the difficult times. Developing ophthalmology research is important with the focus to enhance healthcare.

Information sharing and open access of COVID-19 ophthalmology research is important to allow comparing and validating the scientific conclusions in a different context. There were a large number of submitted manuscripts to peer-reviewed journals around the world during the period of COVID-19, yet a reliable and recognized system to filter and evaluate the huge amount of scientific information is essential to ensure the quality of manuscripts and published data. Healthcare providers faced many challenges with the expanding volume of science and increasing the number of published articles. The gap between theory and practice contributes to part of it. More efforts need to be spent on improving ophthalmology health and awareness of the public regarding emerging and re-emerging of infectious diseases.

Researchers in the field of ophthalmology, big data, applied and engineering field from universities, governmental agencies, and private sectors should unite to fight against COVID19. Besides, in the era of the current pandemic, there is an urgent need to continue research activities while keeping both participants and researchers safe. Remote contact research methodologies such tele-ophthalmology, to save time and cost, improve ease of participation of subjects in studies are important [7]. Besides, effort needs to be put on improving the generalizability of findings, enriching publication that impact translational research positively, and keeping the participants and researchers away from the potential risk of viral transmission. In addition, transparency should be raised to disclose researchers and scientists institutional protocols on conducting COVID-19 research.

\section{Keywords}

Ophthalmology; COVID-19; Research; Publication

\section{References}

1. Au SCL. A surge in eye clinic nonattendance under 2019 novel coronavirus outbreak. Indian J Ophthalmol. 2020;68(5):948.

2. Edgeworth JD, Batra R, Nebbia G, Bisnauthsing K, MacMahon E, Sudhanva M, et al. Translational research in the time of COVID-19-dissolving boundaries. PLoS Pathog. 2020;16(9):e1008898.

3. Au SCL. Revisiting the role of telemedicine under the 2019 novel coronavirus outbreak. Eur J Geriatr Gerontol. 2020;2(1):26-7.

4. Au SC. The transition of academic meetings in 2020. Cancer Res Stat Treat. 2021;4:117-8.

Wu SSK | Volume 2; Issue 2 (2021) | JOAR-2(2)-019 | Letter to Editor

Citation: Au SCL, et al. COVID-19 Publication and the Future of Research. J Ophthalmol Adv Res. 2021;2(2):1-3.

DOI: http://dx.doi.org/10.46889/JOAR.2021.2207 
5. Au SCL. Anti-vascular endothelial growth factor treatment regimens preference by choroidal neovascularization patients under COVID-19. Indian J Ophthalmol. 2020;68(10):2314-5.

6. Sunny CLA. Performing hyperbaric oxygen therapy for central retinal artery occlusion under COVID-19: From myringotomy to rapid viral test. Health Policy Technol. 2021;10(1):29-30.

7. Bunnell BE, Sprague G, Qanungo S, Nichols M, Magruder K, Lauzon S, et al. An exploration of useful telemedicine-based resources for clinical research. Telemed J E Health. 2020;26(1):51-65. 